\title{
Direct-on-Target Microdroplet Growth Assay for Detection of Bacterial Resistance in Positive Blood Cultures
}

\author{
Hao Tang' \\ Rongrong $\mathrm{Li}^{2}$ \\ Huaming $\mathrm{Xu}^{\mathrm{I}}$ \\ Guoping $\mathrm{Lu}^{3}$ \\ Zhen Liu' \\ Wensu Yang' \\ Zhaoxin Xial \\ Yi Zhu' \\ Jilu Shen (D)'
}

'The Fourth Affiliated Hospital of Anhui Medical University Laboratory

Department, Hefei, People's Republic of China; ${ }^{2}$ The First Affiliated Hospital of

Anhui Medical University Laboratory

Department, Hefei, People's Republic of

China; ${ }^{3}$ Laboratory Department of

Fuyang Hospital Affiliated to Anhui

Medical University, Fuyang, Anhui,

236000, People's Republic of China
Correspondence: Jilu Shen

The Fourth Affiliated Hospital of Anhui Medical University Laboratory, No. 100

Huaihai Avenue, Xinzhan District, Hefei,

Anhui Province, 2300I2, People's

Republic of China

Tel +86 I5I 55152963

Email shenjilu@ahmu.edu.cn
Introduction: The recently developed DOT-MGA (direct-on-target microdroplet growth assay) has shown the desirability of direct application of this approach in positive blood cultures and its good performance in detection. This study selected 44 Enterobacteriaceae strains and implemented a DOT-MGA assay on blood cultures to detect their resistance to seven antibiotics. The results of DOT-MGA were compared with the other two antimicrobial susceptibility testing (AST) methods to analyze the detection performance of DOT-MGA.

Methods: We adopted the differential centrifugation to process positive blood-culture (BC). Processed BC broth was directly used for rapid AST using DOT-MGA. Droplets of $6 \mu \mathrm{L}$ with and without antibiotics at the EUCAST breakpoint concentration were spotted in triplicates onto the surface of a MALDI target. The plates were incubated in a wet box for $4 \mathrm{~h}$ before the broth was removed with filter paper. Bruker Biotyper software was used to analyze the test results compared with standard database, and the scores were used to quantify and determine the results.

Results: DOT-MGA results were compared with the direct-from-BC disk-diffusion method and results were reported by broth microdilution method, respectively. The comparison demonstrated a $100 \%$ growth efficiency in DOT-MGA, a 100\% classification consistency for ampicillin, ceftriaxone, and gentamicin, and $>93 \%$ classification consistency for tobramycin, aztreonam, trimethoprim-sulfamethoxazole (TMP-SMX), and ceftazidime.

Discussion: These study results have shown that DOT-MGA is suitable for directly identifying bacterial resistance to positive blood cultures in clinical microbiology laboratories. Furthermore, it is conducive for early diagnosis and treatment of patients with bloodstream infection due to its convenience, time efficiency, and good performance in identifying multiple antibiotic-insensitive bacteria.

Keywords: MALDI-TOF, DOT-MGA, rapid antimicrobial susceptibility testing, blood culture, antibiotic resistance, direct-from-BC disk-diffusion method

\section{Introduction}

Bloodstream infection (BSI) is a major public health burden worldwide, with high mortality. Delayed therapy critically influences patient outcome, and fast and accurate diagnosis decisively improves patients' care. ${ }^{1}$ Identification of the pathogenic microorganisms and fast and accurate antimicrobial susceptibility tests have improved the treatment of patients with sepsis and enabled early treatment that has become particularly important due to increased bacterial resistance to antimicrobials. $^{2,3}$ 
Blood culture (BC) remains the gold standard and firstline tool for diagnosing BSI and sepsis pathogens. ${ }^{4}$ As a standard reference method, antimicrobial susceptibility testing (AST) relies on detecting microbial growth or inhibition in the presence of antibiotics; it features universal detection of resistance, irrespective of the resistance mechanisms. However, it takes about one day to deliver results with currently available standard AST methods, excluding the time spent isolating individual colonies from positive blood cultures. ${ }^{5}$ Alternatively, molecular detection of bacteria and antimicrobial-resistant genes is faster and more sensitive; molecular tests can only detect known resistance mechanisms with a narrow detection scope and high cost. Therefore, it is challenging to widely adopt them in laboratories. Thus, it still takes too much time to produce universal antimicrobial susceptibility reports presently. Furthermore, antimicrobial susceptibility results of patients with sepsis can only be obtained within 24-72 $\mathrm{h}$ after the blood cultures are reported as positive. ${ }^{6}$ By then, patients usually die of a lack of timely diagnosis and treatment.

In the past few years, we have witnessed a dramatic increase in the proportion and absolute number of bacterial pathogens resistant to multiple antibiotics. Multi-drug resistant bacteria are considered an emergent global issue and a major public health problem. ${ }^{7}$ Although the advent of MS has accelerated bacterial identification, it remains exceptionally tricky to achieve rapid AST for patients with microbial infections. The recently developed DOT-MGA allows for rapid universal antimicrobial susceptibility testing using matrix-assisted laser desorption/ionization-time of flight mass spectrometry (MALDI-TOF MS). ${ }^{5,8-10}$ Preliminary tests have shown the desirability of direct application of this approach in positive blood cultures and its good performance in detection. ${ }^{11-13}$

The inoculum size of $5 \times 10^{5} \mathrm{CFU} / \mathrm{mL}$, recommended by the CLSI ${ }^{15}$ and ISO $^{16}$ guidelines as initial inoculum size for AST, is below the lower detection limit by MS. Thus, we used the broth to dilute the bacterial suspension to adjust the bacterial concentration to the initial inoculum size for AST. Then, we incubated the bacteria on a mass spectrometry (MS) target plate to achieve higher bacterial concentrations. Hence, successful detection was reached when bacterial concentrations were above the lower limit of detection by $\mathrm{MS}^{5}$ Additionally, we mixed the bacterial suspension with antibiotics and incubated the mixture on the target plate for $4 \mathrm{~h}$ before submitting the samples for MS.
Thus, antimicrobial resistance was determined based on bacterial growth, ie, bacterial identification and AST could be completed on the same platform. This study selected 44 bacterial strains (including 23 strains of E. coli and 21 strains of Klebsiella pneumoniae) that played a vital role in BSI and mixed a given volume of the bacterial suspension with human blood before injecting the mixture into blood-culture bottles. Differential centrifugation was adopted to process the positive BCs when the blood-culture instrument sounded the alarm. The DOT-MGA was then implemented to detect the resistance of the 44 bacterial strains to the aforementioned seven antibiotics. The results of this study have shown that DOT-MGA is suitable for further development and application in clinical microbiology laboratories due to its good performance in resistance detection.

\section{Materials and Methods}

\section{Bacterial Strains}

Forty-four Enterobacteriaceae strains (including 23 E. coli and 21 Klebsiella pneumoniae strains) isolated from previous daily work were randomly selected and included in this study. All 44 bacterial strains were identified using MALDI-TOF MS (BRUKER, Germany). The minimal inhibitory concentrations (MICs) of the seven antibiotics against all bacterial strains included in this study were determined by the broth microdilution method.

\section{Determination of Minimum Inhibitory Concentration}

Seven antibiotics' minimum inhibitory concentration (MIC) was determined for all isolates using the broth microdilution reference method according to CLSI and ISO guidelines. In brief, bacterial suspensions were prepared in cation-adjusted Mueller-Hinton broth (CA-MHB) to contain approximately $5 \times 10^{5} \mathrm{cfu} / \mathrm{mL}$ as final concentration. Ampicillin, ceftriaxone, gentamicin, tobramycin, aztreonam and ceftazidime were tested at doubling concentrations from 0.125 to $128 \mu \mathrm{g} / \mathrm{mL}$. Trimethoprimsulfamethoxazole (TMP-SMX) was tested in doubling concentrations from 1.25 to $1280 \mu \mathrm{g} / \mathrm{mL}$. The results were read after $18 \pm 2$ hours incubation at $35 \pm 1{ }^{\circ} \mathrm{C}$ (Appendix Table 1). MIC results were interpreted according to the EUCAST breakpoints. Reference strain E. coli ATCC 25922 was used as quality control (QC). 


\section{Inoculation of Blood-Culture Bottles}

Since positive blood-culture specimens were not readily available, blood containing tested bacteria was injected into blood-culture bottles to simulate actual clinical specimens from patients with bacteremia. The blood was collected from people who had not been treated with antibiotics recently. And the blood was sterile blood discarded during routine work. Briefly, bacterial suspension with a standard turbidity of 0.5 McFarland was prepared. Serial dilutions of the standardized bacterial suspension in Mueller-Hinton II broth (cation-adjusted) were performed in steps of $1: 10$ to make a $1: 10^{5}$ dilution. Next, $30 \mu \mathrm{L}$ of bacterial suspension diluted $10^{5}$-times was mixed with $3 \mathrm{~mL}$ of blood to simulate a bacterial concentration of about $10 \mathrm{CFU} / \mathrm{mL}$ commonly observed in patients with bacteremia. ${ }^{11}$ The mixture was then injected into bloodculture bottles and incubated overnight using the automated blood-culture system (Thermo Fisher Scientific). The surface of the blood-culture bottle diaphragm was disinfected with $70 \%$ ethanol before and after injection. ${ }^{12}$

\section{Processing of Positive Blood-Culture Bottles by Differential Centrifugation}

Positive blood-culture bottles were taken from the incubator and immediately processed when the blood-culture instrument sounded the alarm. In this study, we adopted the differential centrifugation to process positive BCs. First, we centrifuged $1.5 \mathrm{~mL}$ of positive bacterial culture suspension with this approach at $2000 \mathrm{r} / \mathrm{min}$ for $5 \mathrm{~min}$ until the blood cells were precipitated. Then, we transferred $1 \mathrm{~mL}$ of supernatant to another test tube and discarded the precipitates and remaining supernatant. Next, the transferred supernatant was centrifuged at $13,000 \mathrm{rpm} /$ min for 2 min before the obtained supernatant was discarded, and the bacterial precipitates were saved. Finally, we resuspended the precipitates in a saline solution and standardized the inoculum for AST.

\section{Direct-from-BC Disk-Diffusion Method}

The updated CLSI M100 S13 document proposed the application of preliminary AST to $\mathrm{BC}$; therefore, we selected six antibiotic disks, including aztreonam, ceftazidime, ceftriaxone, ampicillin (Fisher Scientific), trimethoprim-sulfamethoxazole (TMP-SMX), and tobramycin (Shanghai Guang Rui Biological Technology Co., Ltd) specified in the document. With this approach, we used 20G sterile syringes to draw a well-shaken blood culture and placed four drops of blood culture on the MHA plates. The four drops of blood-culture suspension were then densely streaked with a sterile cotton swab on the surface of MHA plates before the corresponding antibiotic disks were attached. After 16-18 h of incubation, disk-diffusion AST results were reported based on the interpretive standards published in the CLSI M100 document; the inhibition zone diameter breakpoints were also shown (as shown in Appendix Table 2). Additionally, we evaluated the consistency of results generated by DOT-MGA of gentamicin (Fisher Scientific), the broth microdilution method, and direct-from-blood-culture disk-diffusion method. ${ }^{14}$

\section{DOT-MGA}

After positive bacterial cultures were processed by differential centrifugation, DOT-MGA was implemented on the obtained inoculum for fast AST. Ceftazidime, trimethoprim-sulfamethoxazole (TMP-SMX), aztreonam, gentamicin (Shanghai Yuanye Bio-Technology Co., Ltd.), ampicillin, ceftriaxone, and tobramycin (Solarbio) were prepared as dilutions at a given concentration (concentration of the antibiotic is two times the susceptibility breakpoint) in CAMHB. Additionally, the bacterial suspension with a turbidity of $0.5 \mathrm{McF}$ arland was diluted 100 times in CAMHB broth. Next, $100 \mu \mathrm{L}$ of the prepared antibiotic solution was mixed with $100 \mu \mathrm{L}$ bacterial suspension in the wells of the microtitration plates. Hence, the final concentration of the antibiotic reached the susceptibility breakpoint that distinguishes susceptible from non-susceptible Enterobacteriaceae isolates, and the initial inoculation amount of bacteria was $5 \times 10^{5} \mathrm{CFU} / \mathrm{mL}$. The susceptibility breakpoints (concentrations) of ceftazidime, aztreonam, gentamicin, tobramycin, trimethoprimsulfamethoxazole (TMP-SMX), ampicillin, and ceftriaxone were $4 \mu \mathrm{g} / \mathrm{mL}, 4 \mu \mathrm{g} / \mathrm{mL}, 4 \mu \mathrm{g} / \mathrm{mL}, 4 \mu \mathrm{g} / \mathrm{mL}, 2 / 38 \mu \mathrm{g} /$ $\mathrm{mL}, 8 \mu \mathrm{g} / \mathrm{mL}$, and $1 \mu \mathrm{g} / \mathrm{mL}$, respectively.

Additionally, $100 \mu \mathrm{L}$ CAMHB broth was added to $100 \mu \mathrm{L}$ of bacterial suspension in the 96-well microtitration plates as growth controls for each specimen in triplicate to determine the validity of this experiment. After the aforementioned microtitration plate was inoculated, $6 \mu \mathrm{L}$ microdroplets were immediately transferred from the wells of the microtitration plate to a spot on the disposable MALDI-TOF MS target plate. Each specimen containing antibiotics was inoculated into three spots, while the other three spots contained the corresponding growth control of the specimen. The inoculated target plate was then placed inside a plastic transport box (Bruker Daltonik) with 4-mL deionized water added to the 
bottom of the box so that the transport box could be used as a wet box. After the wet box was incubated at $35^{\circ} \mathrm{C} \pm 1^{\circ} \mathrm{C}$ for 4 $\mathrm{h}$, the broth was removed from the target plate with filter paper.

After the target plate was completely dry, the matrix (IVD Matrix HCCA-portioned, Bruker Daltonik) was treated with 1 $\mu \mathrm{L}$ MS samples to overlay the target spots. The MALDI-TOF MS spectra were manually collected and evaluated using standard MALDI-Biotyper 3 software (Bruker Daltonik). In this study, Bruker Biotyper software was used to analyze the test results compared with standard database, and the scores were used to quantify and determine the results. For the nonantibiotic growth controls, the assay was considered effective if an identification score $\geq 1.7$ was achieved. For specimens containing the tested antibiotics, successful identification (score $\geq 1.7$ ) was interpreted as non-susceptible (intermediate or resistant), while no identification (score $<1.7$ ) was defined as susceptible. In this study, intermediate and resistant strains were classified as non-susceptible strains.

\section{Data Analysis}

The final results of DOT-MGA were compared with those of the direct-from-blood-culture disk-diffusion method and the broth microdilution method. The efficiency, sensitivity, specificity, and classification consistency of the method were calculated, respectively.

\section{Results}

\section{The Detection Performance of DOT-MGA}

In previous target-plate microdroplet growth assays, $6-\mu \mathrm{L}$ droplets have proven conducive to achieving the best detection performance. At the early stage of the study, we conducted a preliminary examination on the impact of droplet size on the detection results. An excessively large droplet is prone to overflow and cross-contamination, while an excessively small droplet might lead to poor bacterial growth and lower growth efficiency. Meanwhile, we explored the impact of incubation time on identification results at the early stage of the study and found that after 4 $\mathrm{h}$ of incubation, efficient growth in the control wells and high detection performance were achieved. However, a longer incubation duration did not significantly enhance the detection performance; less than $4 \mathrm{~h}$ incubation time resulted in lower growth efficiency. Therefore, we used a $6-\mu \mathrm{L}$ droplet volume with $4 \mathrm{~h}$ of incubation for assays in subsequent studies. This study achieved efficient growth for the 44 tested bacterial strains (ie, 100\% growth efficiency), which indicated that all assays were effective. MALDI-TOF MS data were evaluated using a simple algorithm using the MALDI-BIOTYPER software. As a result, the susceptible isolates could be rapidly and accurately distinguished from non-susceptible isolates. Successful species identification (score $\geq 1.7$ ) was interpreted as a non-susceptible result (ie, intermediate or resistant), while no identification (score $<1.7)$ was defined as susceptible. The final results of DOTMGA are shown in Appendix Table 3.

The broth microdilution method is the gold standard for AST, and it can determine the MIC. However, the operation of this method is complicated and timeconsuming, which makes it difficult to apply to routine laboratory operations. The classification consistency between the broth microdilution method and DOT-MGA is above $95 \%$. Based on the broth microdilution method, we calculated the specificity and sensitivity of DOT-MGA, and the results are shown in Table 1. Direct-from-BC diskdiffusion method can directly use the original blood culture samples for AST. However, it takes 16-18 hours, which is significantly longer than DOT-MGA. The classification consistency between Direct-from-BC diskdiffusion method and DOT-MGA is above 93\%. At the same time, we calculated the specificity and sensitivity of

Table I Performance of DOT-MGA on Identifying Non-Susceptible Strains with Results Generated Using the Broth Microdilution Method as the Benchmark

\begin{tabular}{|l|c|c|c|c|}
\hline Antibacterial Agents & Efficiency (100\%) & Sensitivity (100\%) & Specificity (100\%) & Classification Consistency (100\%) \\
\hline Ampicillin & 100 & 100 & 100 & 100 \\
Ceftriaxone & 100 & 100 & 100 & 100 \\
Tobramycin & 100 & 88.9 & 100 & 95.5 \\
Ceftazidime & 100 & 100 & 100 & 100 \\
Aztreonam & 100 & 100 & 100 & 100 \\
TMP-SMX & 100 & 100 & 91.3 & 95.5 \\
gentamicin & 100 & 100 & 100 \\
\hline
\end{tabular}


Table 2 Performance of DOT-MGA on Identifying Non-Susceptible Strains with Results Generated Using Direct-from-Blood-Culture Disk-Diffusion Method as the Benchmark

\begin{tabular}{|l|c|c|c|c|}
\hline Antibacterial Agents & Efficiency (100\%) & Sensitivity (100\%) & Specificity (100\%) & Classification Consistency (100\%) \\
\hline Ampicillin & 100 & 100 & 100 & 100 \\
Ceftriaxone & 100 & 100 & 100 & 100 \\
Tobramycin & 100 & 88.9 & 100 & 95.5 \\
Ceftazidime & 100 & 94.1 & 100 & 97.7 \\
Aztreonam & 100 & 94.1 & 100 & 97.7 \\
TMP-SMX & 100 & 95.5 & 90.9 & 93.2 \\
Gentamicin & 100 & 100 & 100 & 100 \\
\hline
\end{tabular}

DOT-MGA based on the Direct-from-BC disc diffusion method. The results are shown in Table 2 .

\section{Discussion}

Over the past decade, MALDI-TOF MS in clinical microbiology has brought great convenience and notably improved accuracy and speed for clinical microbiology diagnostics. However, progress brought by MALDI-TOF MS is mainly limited to strain identification, and rapid AST remains a challenge that needs to be urgently tackled. Although the latest report published by CLSI specifies the application of direct-from-blood-culture disk-diffusion method in rapid AST on BC, it still takes $18 \mathrm{~h}$ before AST results can be obtained. This means that AST reports of patients with bacteremia can only be obtained on the second day after the blood cultures are reported as positive. This issue will undoubtedly lead to inappropriate medication use and delayed therapy.

This study adopted DOT-MGA on blood cultures based on the principle of the broth microdilution method. The difference is that we incubated the tested bacteria on the MS target plate and determined bacterial growth at the breakpoint concentration based on MS identification scores. Compared with the broth microdilution method, DOT-MGA also further eliminates the ambiguity in test results caused by the subjectivity of naked-eye observations. At the early stage of the study, we tried spotting the bacterial suspension incubated on the microtitration plate for $4 \mathrm{~h}$ directly on the target plate for identification. However, the results were unstable, possibly because too much broth residue interfered with the performance of the mass spectrometer. Hence, in this study, we gently blotted the microdroplets with cheap and easily available Cleanroom Wipers (Dongguan Jia Chong Purification Technology Co., Ltd) to remove the broth through capillary action. However, such gentle "contact" with the microdroplets would not remove the bacteria, at least not to the degree of interfering with the performance of MS analysis. During the incubation process, biofilms were possibly formed by bacteria adhered to the surface of the MALDI plate, ${ }^{13}$ which helped in the successful separation of broth from the target plate.

After positive blood cultures were treated with differential centrifugation, bacterial resistance was directly determined by target-plate microdroplet growth assays. With this approach, the specimens were easily handled, and AST results were obtained in only $5 \mathrm{~h}$ after blood cultures were reported as positive. Positive blood cultures processed with the lysis centrifugation technique mentioned in other reports might leave a cleaner background on the MS target plate; however, lysing reagents are expensive, and the cost of a single assay is high. DOTMGA is notably superior to the broth microdilution method and direct-from-blood-culture disk-diffusion method in terms of speed and easy operation. The final results demonstrated good detection performance of this approach, with $>93 \%$ result consistency generated by DOT-MGA and the other two testing methods for all seven antibiotics. For the false-positive and falsenegative results of individual strains, the MIC may be too close to the sensitive breakpoint concentration. Conclusively, the approach adopted in this study offers the advantages of simple and easy operation, high efficiency, low cost, and high consistency with reference methods. Once the optimal laboratory workflow is confirmed, AST results can be generated within the same working shift in microbiology laboratories and delivered to clinicians. This process accelerates the diagnosis and treatment of BSI, enables early effective antimicrobial treatment that might lower mortality rates, and provides considerable support for treatment decision-making and patient management. 
Moreover, although the executive standards of the direct-from-blood-culture disk-diffusion method for gentamicin are not specified in the CLSI M100 S13 document, our study showed a $100 \%$ consistency of results generated by DOT-MGA, the broth microdilution method, and direct-from-blood-culture disk-diffusion methods. Due to insufficient energy, we only evaluated the accuracy of target-plate microdroplet growth assays on blood cultures for the aforementioned seven antibiotics. In addition, we only selected and tested E. coli and Klebsiella pneumoniae in this study; however, other researchers have suggested that this approach also featured high accuracy in the AST of Staphylococcus and Pseudomonas aeruginosa. ${ }^{5,12} \mathrm{We}$ expect multiple antibiotics to be deposited on the disposable MS target plate in the "lyophilized powder" form so AST results can be obtained within several hours after a bacterial suspension is applied to the plate. Easy-to-use disposable target plates do not require cleaning and can prevent contamination of the specimens, which is crucial for AST. Notably, this approach integrates bacterial identification and AST into the same platform, which largely reduces deviations in AST results caused by different operators involved in the two independent tests.

Summarily, this study has proven the feasibility and accuracy of DOT-MGA directly on positive blood cultures for rapid AST, which is conducive for inhibiting antimicrobial-resistant microorganisms in clinical practice and epidemiology. However, further studies need to be conducted to standardize and optimize test conditions and evaluation criteria.

\section{Ethical Approval}

This study involved anonymous use of redundant, abandoned patient blood, which is part of the standard treatment agreements with patients in our hospital. This research does not affect patients' health and privacy. Ethics approval was obtained from the medical ethics committee of Anhui Medical University with the following reference number: LLSC20210802.

\section{Author Contributions}

All authors made substantial contributions to conception and design, acquisition of data, or analysis and interpretation of data; took part in drafting the article or revising it critically for important intellectual content; agreed to submit to the current journal; gave final approval for the version to be published; and agreed to be accountable for all aspects of the work.

\section{Funding}

This work was funded through a grant from Anhui Provincial Department of Education for university cooperative research and public health collaborative innovation project in Anhui Province in 2020 (Grant No. GXXT2020-016) and a grant from Anhui Provincial Health Commission for key scientific research projects in 2021 (Grant No. AHWJ2021a011).

\section{Disclosure}

The authors report no conflicts of interest in this work.

\section{References}

1. Lamy B, Sundqvist M, Idelevich EA. Bloodstream infections Standard and progress in pathogen diagnostics. Clin Microbiol Infect. 2020;26(2):142-150. doi:10.1016/j.cmi.2019.11.017

2. Pliakos EE, Andreatos N, Shehadeh F, Ziakas PD, Mylonakis E. The cost-effectiveness of rapid diagnostic testing for the diagnosis of bloodstream infections with or without antimicrobial stewardship. Clin Microbiol Rev. 2018;31(3). doi:10.1128/ CMR.00095-17

3. Barenfanger J, Graham DR, Kolluri L, et al. Decreased mortality associated with prompt gram staining of blood cultures. Am J Clin Pathol. 2008;130(6):870-876. doi:10.1309/AJCPVMDQU2 ZJDPBL

4. Rhodes A, Evans LE, Alhazzani W, et al. Surviving sepsis campaign: international guidelines for management of sepsis and septic shock: 2016. Intensive Care Med. 2017;43(3):304-377.

5. Idelevich EA, Sparbier K, Kostrzewa M, Becker K. Rapid detection of antibiotic resistance by MALDI-TOF mass spectrometry using a novel direct-on-target microdroplet growth assay. Clin Microbiol Infect. 2018;24(7):738-743. doi:10.1016/j. cmi.2017.10.016

6. van Belkum A, Bachmann TT, Lüdke G, et al. Developmental roadmap for antimicrobial susceptibility testing systems. Nat Rev Microbiol. 2019;17(1):51-62. doi:10.1038/s41579-018-0098-9

7. Roca I, Akova M, Baquero F, et al. The global threat of antimicrobial resistance: science for intervention. New Microbes New Infect. 2015;6:22-29. doi:10.1016/j.nmni.2015.02.007

8. Li R, Tang H, Xu H, Ren Y, Li S, Shen J. Direct-on-target microdroplet growth assay applications for clinical antimicrobial susceptibility testing. Infect Drug Resist. 2021;14:1423-1425. doi:10.2147/ IDR.S303187

9. Correa-Martínez CL, Idelevich EA, Sparbier K, Kuczius T, Kostrzewa M, Becker K. Development of a MALDI-TOF MS-based screening panel for accelerated differential detection of carbapenemases in Enterobacterales using the direct-on-target microdroplet growth assay. Sci Rep. 2020;10(1):4988. doi:10.1038/s41598020-61890-7

10. Neonakis IK, Spandidos DA. MALDI-TOF mass spectrometry-based direct-on-target microdroplet growth assay: a novel assay for susceptibility testing and beyond. Future Microbiol. 2019;14(9):729-731. doi:10.2217/fmb-2019-0106

11. Idelevich EA, Storck LM, Sparbier K, Drews O, Kostrzewa M, Becker K. Rapid direct susceptibility testing from positive blood cultures by the matrix-assisted laser desorption ionization-time of flight mass spectrometry-based direct-on-target microdroplet growth assay. J Clin Microbiol. 2018;56(10):e00913-e00918. doi:10.1128/ JCM.00913-18 
12. Nix ID, Idelevich EA, Storck LM, et al. Detection of methicillin resistance in Staphylococcus aureus from agar cultures and directly from positive blood cultures using MALDI-TOF mass spectrometry-based direct-on-target microdroplet growth assay. Front Microbiol. 2020;11:232. doi:10.3389/fmicb.2020.00232

13. Idelevich EA, Becker K. MALDI-TOF mass spectrometry for antimicrobial susceptibility testing. J Clin Microbiol. 2021;Jcm0181419. doi:10.1128/JCM.01814-19

14. Chandrasekaran S, Abbott A, Campeau S, et al. Direct-from-bloodculture disk diffusion to determine antimicrobial susceptibility of gram-negative bacteria: preliminary report from the clinical and laboratory standards institute methods development and standardization working group. J Clin Microbiol. 2018;56(3):e01678-e01717. doi:10.1128/JCM.01678-17.
15. CLSI. Methods for Dilution Antimicrobial Susceptibility Tests for Bacteria That Grow Aerobically; Approved Standard. 10th ed. M07eA10. Wayne, PA: Clinical Laboratory Standards Institute; 2015.

16. ISO. 20776-1. Clinical laboratory testing and in vitro diagnostic test systems - susceptibility testing of infectious agents and evaluation of performance of antimicrobial susceptibility test devices - Part 1: reference method for testing the in vitro activity of antimicrobial agents against rapidly growing aerobic bacteria involved in infectious diseases. Geneva, Switzerland: International Organization for Standardization; 2006.

\section{Publish your work in this journal}

Infection and Drug Resistance is an international, peer-reviewed openaccess journal that focuses on the optimal treatment of infection (bacterial, fungal and viral) and the development and institution of preventive strategies to minimize the development and spread of resistance. The journal is specifically concerned with the epidemiology of antibiotic resistance and the mechanisms of resistance development and diffusion in both hospitals and the community. The manuscript management system is completely online and includes a very quick and fair peerreview system, which is all easy to use. Visit http://www.dovepress.com/ testimonials.php to read real quotes from published authors. 